\title{
Myosin Binding Protein-C Slow: An Intricate Subfamily of Proteins
}

\author{
Maegen A. Ackermann and Aikaterini Kontrogianni-Konstantopoulos \\ Department of Biochemistry and Molecular Biology, School of Medicine, University of Maryland, Baltimore, MD 21201, USA \\ Correspondence should be addressed to Aikaterini Kontrogianni-Konstantopoulos, akons001@umaryland.edu
}

Received 7 December 2009; Accepted 1 February 2010

Academic Editor: Henk L. M. Granzier

Copyright ( $\odot 2010$ M. A. Ackermann and A. Kontrogianni-Konstantopoulos. This is an open access article distributed under the Creative Commons Attribution License, which permits unrestricted use, distribution, and reproduction in any medium, provided the original work is properly cited.

\begin{abstract}
Myosin binding protein $\mathrm{C}$ (MyBP-C) consists of a family of thick filament associated proteins. Three isoforms of MyBP-C exist in striated muscles: cardiac, slow skeletal, and fast skeletal. To date, most studies have focused on the cardiac form, due to its direct involvement in the development of hypertrophic cardiomyopathy. Here we focus on the slow skeletal form, discuss past and current literature, and present evidence to support that: (i) MyBP-C slow comprises a subfamily of four proteins, resulting from complex alternative shuffling of the single MyBP-C slow gene, (ii) the four MyBP-C slow isoforms are expressed in variable amounts in different skeletal muscles, (iii) at least one MyBP-C slow isoform is preferentially found at the periphery of $M$-bands and (iv) the MyBP-C slow subfamily may play important roles in the assembly and stabilization of sarcomeric $M$ - and $A$-bands and regulate the contractile properties of the actomyosin filaments.
\end{abstract}

\section{Introduction}

Myofibrils, the workhorses of skeletal muscle, consist of interdigitating thick and thin filaments, and their associated membrane systems [1]. Muscle contraction and relaxation is mediated by the sliding of thick myosin filaments past thin actin filaments, under the strict regulation of $\mathrm{Ca}^{2+}$ release and reuptake via the sarcoplasmic reticulum (SR) [2]. In addition to housing the basic thick and thin filaments, the sarcomere also contains several accessory proteins that are involved in the assembly, maintenance, and regulation of contractile activity [1]. Myosin Binding Protein-C (MyBPC) comprises a family of accessory proteins that contributes to the assembly and stabilization of thick filaments, and regulates the formation of cross-bridges between myosin and actin by interacting directly with both filamentous systems (as reviewed in [3]).

MyBP-C was originally identified from mammalian skeletal muscle as an impurity of isolated myosin. Using SDSpolyacrylamide gel electrophoresis (SDS-PAGE), Star and Offer were the first to separate a number of unidentified myosin-associated proteins that were consistently found in preparations of purified myosin [4]. MyBP-C, originally termed C-protein for its location on SDS-PAGE as band $C$, was further characterized as a myosin binding protein of $\sim 140 \mathrm{kDa}$ using a combination of biochemical methods, ranging from gel filtration, to ammonium sulfate fractionation and single molecule electron microscopy [4-6]. The location of MyBP-C at striped intervals within the C-zone of the A-band of skeletal muscle was first observed with Xray diffraction and immunoelectron microscopy [7], further supporting its association with the thick myosin filaments. Subsequent studies revealed that MyBP-C is arranged along the length of the $A$-band in 7-9 transverse stripes that are $\sim 43 \mathrm{~nm}$ apart, with $\sim 2-4$ molecules of MyBP-C associating with each myosin cross-bridge [8-11].

The family of MyBP-C contains three isoforms: cardiac, slow skeletal, and fast skeletal, which are encoded by separate genes; in humans, these map to chromosomes 11, 12, and 19 , respectively $[12,13]$. The different isoforms have been cloned and sequenced from various species, including human, chicken, rabbit and mouse, allowing a thorough comparison of their molecular composition and primary sequence [13-16]. An $\sim 65 \%$ identity is shared among the individual isoforms across species, while an $\sim 50 \%$ homology is shared among the human cardiac, slow and fast forms. 
The core structure of MyBP-C is composed of seven immunoglobulin (Ig) domains and three fibronectin type III (Fn-III) repeats, numbered from the $\mathrm{NH}_{2}$-terminus as $\mathrm{C} 1-\mathrm{C} 10$ [17]. The $\mathrm{C} 1$ domain is flanked by two unique motifs, one enriched in proline and alanine residues, termed proline/alanine rich motif and a conserved linker, referred to as MyBP-C motif. Notably, the cardiac isoform possesses three additional features, which are absent from the skeletal forms of the protein. These include an Ig domain at the extreme $\mathrm{NH}_{2}$-terminus of the molecule, termed $\mathrm{C} 0$, a unique 9-residues long insertion within the MyBP-C motif containing phosphorylation sites necessary for the protein's regulatory role in contraction, and a 28-amino acids long loop in the middle of the $\mathrm{C} 5$ domain $[12,16]$.

In mammals, cardiac MyBP-C is expressed early in development, along with titin and myosin $[18,19]$. The skeletal isoforms of MyBP-C, however, are detected later in development, after the expression of titin and myosin, with the expression of slow MyBP-C preceding that of fast $[18,20]$. By contrast, in chicken skeletal muscles the slow and fast isoforms appear concurrently at the end of late embryogenesis [21]. As development proceeds, though, the amounts of the slow form diminish, while the expression of the fast form persists through adulthood [21]. The fast and slow skeletal isoforms have been shown to coexpress in the same muscle type and can even coexist in the same sarcomere; expression of the cardiac isoform, however, is restricted to the developing and mature heart [15, 18, 22-24]. Interestingly, a recent study demonstrated that the expression of MyBP-C slow is not restricted to skeletal muscle but is also evidenced in the right atrium and interatrial septum of adult mammalian cardiac muscle [25].

To date, much of our knowledge on the molecular properties and functional activities of MyBP-C originates from the numerous studies that focus on the cardiac isoform. The reader is referred to excellent reviews discussing the structure of cardiac MyBP-C, its key roles in maintaining the normal structure of thick filaments and regulating crossbridge cycling, and its involvement in the development of hypertrophic cardiomyopathy $[3,26,27]$. Here, we will focus on the slow form(s) of MyBP-C found in skeletal muscles. We will review past and current literature, discuss its role in the organization and stabilization of thick filaments, and provide evidence that MyBP-C slow comprises a subfamily of four alternatively spliced proteins that are expressed in variable amounts in slow and fast twitch skeletal muscles.

\section{Materials and Methods}

2.1. Reverse Transcription-Polymerase Chain Reaction. Total RNA was isolated with Trizol reagent (Invitrogen, Carlsbad, CA) from P1 rat skeletal myotubes cultured for seven days and from adult rat extensor digitorum longus (EDL), flexor digitorum brevis (FDB), tibialis anterior (TA), gastrocnemius (gastroc), quadriceps (quad), and soleus muscles. Aliquots containing $\sim 5 \mu \mathrm{g}$ of RNA were reverse transcribed using the Superscript First Strand Synthesis System for RT-PCR (Invitrogen, Carlsbad, CA) following the manufacturer's instructions. PCR amplification of MyBPC slow transcripts was performed with primer sets that flanked each of the three insertions; for the $\mathrm{NH}_{2}$-terminal insert: Forward-1 (F1): 5' CCAGAACCCACTAAGAAAG 3' and Reverse-1 (R1): 5' GATCCTCGAGGTGCACTT CAAGATCAA $3^{\prime}$, for the insert within Ig7: Forward-2 (F2): $5^{\prime}$ GATCGAATTCAGCCC TCCTACTCTT $3^{\prime}$ and Reverse2 (R2): $5^{\prime}$ GATCCTCGAGGGGCTCGCTGGCACCA $3^{\prime}$ and for the COOH-terminal insert: Forward-3 (F3): $5^{\prime}$ CACCCATGTTTACTCAACCCT 3' and Reverse-3 (R-3) 5' GTGACAATATACATTGAA $3^{\prime}$. To amplify the $\mathrm{COOH}$-terminal insert from EDL and TA muscles, it was necessary to reamplify $0.5 \mu \mathrm{L}$ of the original PCR for an additional 30 cycles for a total of 80 cycles. All other PCRs were carried out for 50 cycles. PCR products were analyzed by electrophoresis in $1 \%$ agarose gels and their authenticity was verified by sequence analysis.

\subsection{Generation of Protein Lysates from P1 Myotubes and Adult} Rat Muscle. Homogenates of P1 myotubes cultured for seven days as well as of EDL, FDB, TA, gastrocnemious, quadriceps and soleus muscles of adult Sprague-Dawley rats (ZivicMiller Laboratories, Zelienople, PA) were prepared at RT with a Brinkman Polytron homogenizer at setting 3 (VWR, West Chester, PA) in $10 \mathrm{mM} \mathrm{NaPO}_{4}, \mathrm{pH} 7.2,2 \mathrm{mM}$ EDTA, $10 \mathrm{mM} \mathrm{NaN}_{3}, 120 \mathrm{mM} \mathrm{NaCl}, 0.5 \%$ deoxycholate, $0.5 \% \mathrm{NP}$ 40 , supplemented with Complete protease inhibitors (Roche, Indianapolis, IN). Each sample $(\sim 60 \mu \mathrm{g})$ was solubilized in $4 \mathrm{xNuPAGE}$ reducing sample buffer (Invitrogen, Carlsbad, $\mathrm{CA})$ at $90^{\circ} \mathrm{C}$ for 5 minutes, fractionated by $4-12 \%$ SDSPAGE BisTris gel using MES running buffer (Invitrogen, Carlsbad, CA), transferred to nitrocellulose and probed with an antibody that recognizes the slow forms of MyBP-C $(300 \mathrm{ng} / \mathrm{mL}$, Abnova) or with an antibody that specifically recognizes the $\mathrm{COOH}$-terminal insert [28]. After incubation with the appropriate secondary antibodies, immunoreactive bands were visualized with a chemiluminescence detection kit (Tropix, Bedford, MA).

2.3. Immuno-Electron Microscopy. Immuno-electron microscopy was performed as previously described with minor modifications [29, 30]. Briefly, adult mouse FDB skeletal muscle was fixed both in situ, via whole animal perfusionfixation and ex vivo with $2 \%$ paraformaldehyde in PBS. Samples were snap-frozen in a slush of liquid $\mathrm{N}_{2}$, cryosectioned $\left(\sim 20 \mu \mathrm{m}\right.$ thick), and incubated overnight at $4^{\circ} \mathrm{C}$ with the antibody that specifically recognizes the $\mathrm{COOH}$ terminal insert of MyBP-C slow. Samples were incubated with secondary goat antirabbit IgG adsorbed on $1 \mathrm{~nm}$ gold particles (Nanoprobes Incorporated; Yaphank, NY) and subsequently with fluorescein-conjugated antigoat IgG (Jackson ImmunoResearch Laboratories Inc., West Grove, PA). Samples were enhanced with silver (HiQ Silver Kit, Nanoprobes) for 5 minutes, fixed overnight at $4^{\circ} \mathrm{C}$ in $2 \%$ gluteraldehyde and $5 \mathrm{mg} / \mathrm{mL}$ tannic acid in $0.2 \mathrm{M}$ cacodylate buffer and postfixed with $50 \mathrm{mM}$ acetate buffer $1 \%$ osmium tetroxide. They were further stained en bloc for 2 hours with $1 \%$ uranyl acetate in $65 \%$ ethanol, 
dehydrated, and embedded in araldite (Electron Microscopy Sciences, Fort Washington, PA). Ultrathin $(60-90 \mathrm{~nm}) \mathrm{sec}-$ tions were prepared with an MT5000 ultramicrotome (LKB instruments Inc., Gaithersburg, MD), mounted on grids, labeled with $1 \%$ uranyl acetate followed by Reynolds lead citrate, and examined with a Philips-201 electron microscope.

\section{Results and Discussion}

3.1. MyBP-C Slow: A Subfamily of Proteins. To date, four different MyBP-C slow transcripts have been identified in human skeletal muscle referred to as variants 1-4 (Figure 1; accession numbers NM_002465, NM_206819, NM_206820, and NM_206821, respectively). The four variants differ from one another at three regions, due to alternative splicing events that result in inclusion of exons 3 and 4 in the proline/alanine-rich motif, exon 23 in the $\operatorname{Ig} 7$ domain and exon 31 at the extreme $\mathrm{COOH}$-terminus (Figure 1(a)); these encode novel sequences of 25 (Figure 1(b)), 18 (Figure 1(c)), and 26 (Figure 1(d)) amino acids, respectively. Analysis of the primary sequence of the four MyBP-C slow variants indicated that variants 1 and 2 contain the $\mathrm{NH}_{2}$-terminal insertion located in the proline/alanine rich motif, variant 3 carries the insertion within domain Ig7, while variant 1 also contains the unique $\mathrm{COOH}$-terminal region (Figure $1(\mathrm{a})$ ). Notably, variant 3 is the prototypical human isoform of MyBP-C slow that was characterized by Furst and colleagues in 1992 [14].

To study the relative expression of the four MyBP-C slow transcripts in different rat skeletal muscles, we used RT-PCR analysis to amplify the unique regions described above. To this end, we prepared cDNAs from a panel of adult and developing rat skeletal muscles that contained distinct compositions of slow and fast twitch skeletal myofibers. These included extensor digitorum longus (EDL; 90:10, fast: slow; [31,32]), flexor digitorum brevis (FDB; $~ 80: 20$, fast: slow; [33]), tibialis anterior (TA; $\sim 70: 30$, fast: slow; [34]), gastrocnemius (gastroc; $\sim 40: 60$, fast: slow; [35]), quadriceps (quad; $\sim 60: 40$, fast : slow; [36]), soleus $(20: 80$, fast : slow, [35]), and hindlimb skeletal myotubes of postnatal day 1 (P1) rat pups (Figure 2). Primer sets were designed to flank each of the three novel insertions (Figures 2(a)2(c), cartoons in the upper left corner). Amplification of two PCR products with distinct sizes within each reaction indicated the presence of a mixed population of transcripts that contained (larger size product) and lacked (smaller size product) the respective insertion. On the contrary, amplification of one PCR product indicated the presence of a homogeneous population of transcripts that either included or excluded the corresponding insertion, depending on its size. Accordingly, PCR products that carry the $\mathrm{NH}_{2}$ terminal, Ig7 and COOH-terminal inserts would be $\sim 600$, $\sim 310$, and $\sim 350$ nucleotides long, respectively, whereas PCR products that lack them would be $\sim 530, \sim 260$, and $\sim 290$ nucleotides long, respectively.

All skeletal muscles examined, independent of their fiber type composition, contained sufficient amounts of MyBP-C slow transcripts to be amplified by conventional RT-PCR. Figure 2(a) shows the results following amplification of the $\mathrm{NH}_{2}$-terminal insertion located within the proline/alanine rich motif. All muscle samples express MyBP-C slow transcripts that include the $\mathrm{NH}_{2}$-terminal insert, albeit to varying degrees, as seen by the presence of an $\sim 600 \mathrm{nts}$ amplicon (Figure 2(a), upper band). This finding suggested that all muscles tested express variants 1 and/or 2, with EDL, TA, and soleus containing the highest amounts. Notably, all seven muscles also contain different amounts of transcripts that lack the $\mathrm{NH}_{2}$-terminal insert, as shown by the presence of an $\sim 530 \mathrm{nts}$ product, indicating that they also express variants 3 and/or 4, with FDB showing the highest levels (Figure 2(a), lower band). Contrary to EDL, TA, soleus, and FDB that appear to preferentially contain MyBP-C slow variants that either include (EDL, TA and soleus) or exclude (FDB) the $\mathrm{NH}_{2}$-terminal insert, gastroc, quad, and P1 myotubes show similar amounts of both amplification products, suggesting that transcripts possessing and lacking the $\mathrm{NH}_{2}$-terminal insert may exist in similar levels within these muscles (Figure 2(a) lanes 4-5 and 7).

Next, we extended our analysis to the second novel insertion located within Ig7. As before, our primers set was designed to amplify MyBP-C slow variants that contained or skipped the Ig7 insert. Only soleus and P1 myotubes possess transcripts that include the unique insertion within Ig7, as indicated by the presence of an $\sim 310 \mathrm{nts}$ band (Figure 2(b), lanes 6 and 7, upper band), corresponding to variant 3 . As expected, all skeletal muscles tested contained transcripts that lack the Ig7 insertion, as shown by the amplification of an $\sim 260$ nts product, representing variants 1, 2 and/or 4 . Thus, variant 3 is restricted to soleus muscle and developing myotubes.

Last, we generated the appropriate primers to amplify the region flanking the $\mathrm{COOH}$-terminal insert of MyBP$\mathrm{C}$ slow. With the exception of quadriceps muscle, all other muscles tested, contained an amplification product of $\sim 350$ nucleotides, that includes the novel $\mathrm{COOH}$-terminal insert detected only in variant 1 , with soleus and FDB showing the highest amounts (Figure 2(c), lanes 6 and 2, upper band). A second amplification product of $\sim 290$ nucleotides was also detected in all seven muscles, which lacks the unique $\mathrm{COOH}$-terminal insertion, present in variant 1 , but includes the common $\mathrm{COOH}$-terminal region shared by variants 2,3 , and 4 (Figure 2(c), lower band).

The RT-PCR data is summarized in Table 1. Taken together, our results suggest that: (i) mRNA encoding MyBP$\mathrm{C}$ slow is present in all skeletal muscles examined, regardless of fiber type composition or age, and (ii) complex alternative shuffling of the single MyBP-C slow gene in the various muscles results in the differential expression of the four MyBP-C slow isoforms.

To correlate the mRNA expression of the transcripts that contain or lack the three novel insertions, as seen by the RT-PCR, to the expression of the proteins that they encode, we used western blot analysis. Homogenates from EDL, FDB, TA, gastroc, quad, soleus, and P1 skeletal myotubes were separated on $4-12 \%$ gradient gel, which our laboratory has previously shown to provide optimal 


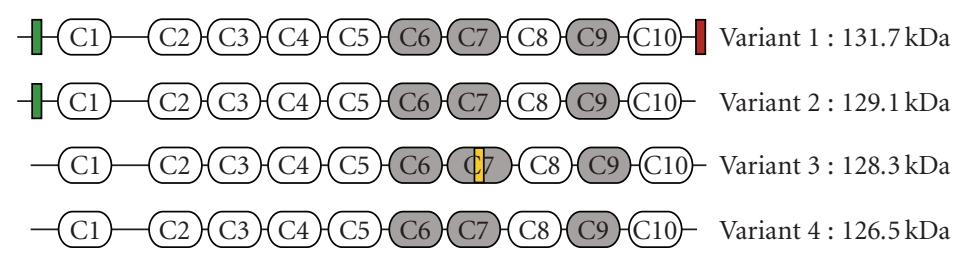

(a)

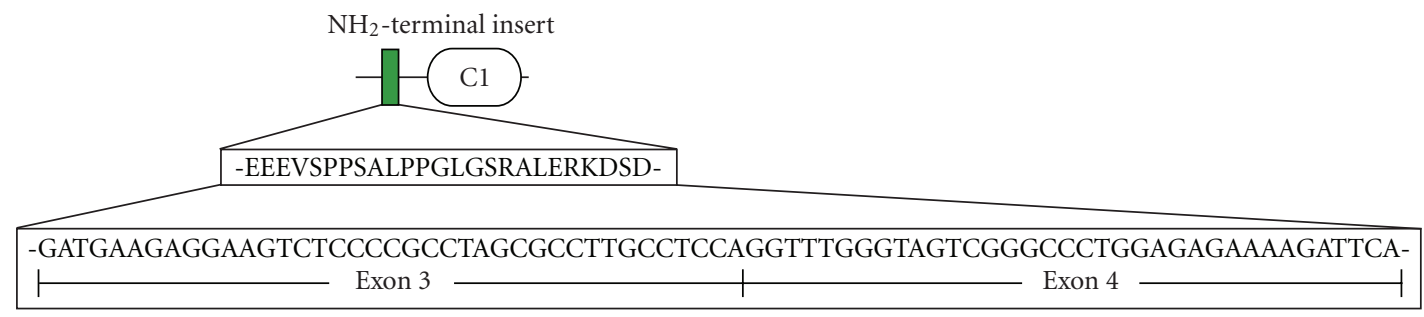

(b)

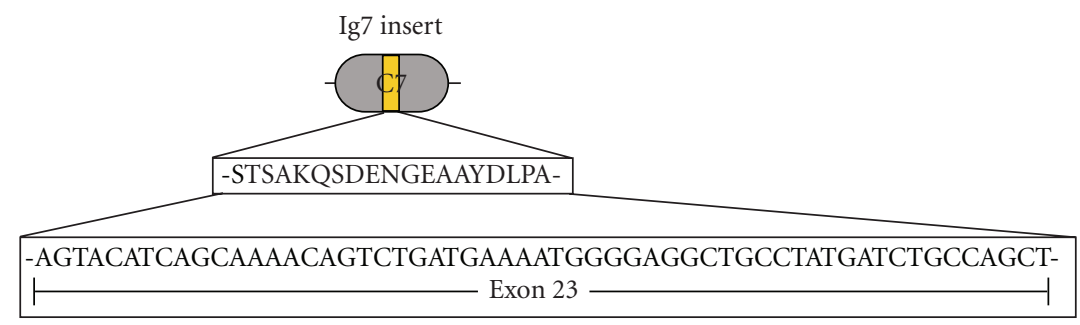

(c)

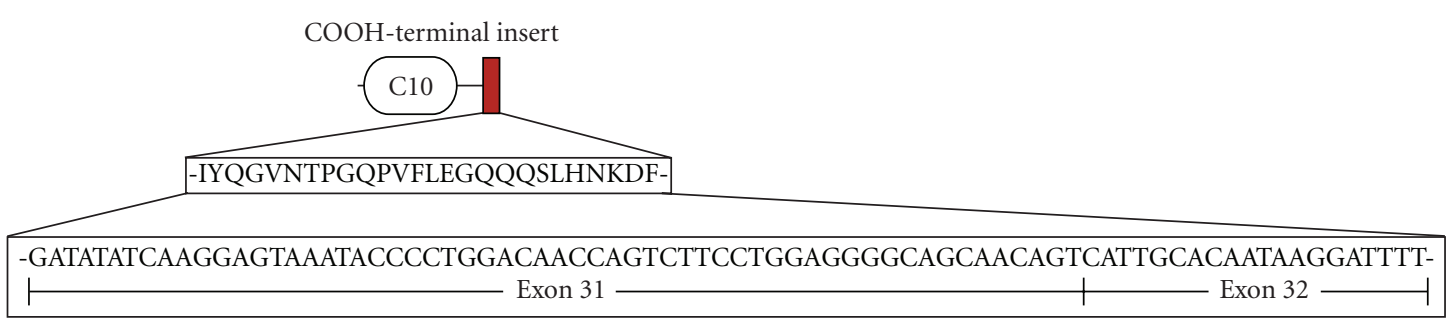

(d)

FIGURE 1: (a): Schematic representation of MyBP-C slow variants 1-4, showing their common structural motifs and novel insertions; white and grey ovals represent Ig and FN-III domains, respectively, while green, yellow, and red rectangles correspond to the $\mathrm{NH}_{2}$-terminal, Ig7, and $\mathrm{COOH}$-terminal inserts, respectively. The $\mathrm{NH}_{2}$-terminal insert is present in variants 1 and 2, while the Ig7 and $\mathrm{COOH}$-terminal inserts are present in variants 3 and 1, respectively. Variant 4 does not contain any of the three inserts. (b-d): Complex alternative splicing events result in the inclusion of exons 3 and 4, exon 23 and exon 31 that encode the $\mathrm{NH}_{2}$-terminal (b), Ig7 (c), and COOH-terminal (d) novel insertions. The amino acid and nucleotide compositions of the three insertions are shown.

separation of the MyBP-C slow isoforms (see Section 2, and [28]). Homogenates were probed with a commercial antibody that recognizes a region within domain C5 common to all MyBP-C slow variants (Figure 3(a)). We were able to resolve at least 3 immunoreactive bands based on their distinct electrophoretic mobilities. The top band may represent variant $1(\sim 132 \mathrm{kDa}$, Figure $3(\mathrm{a})$, top panel, marked with a blue dot), the middle band may correspond to variants 2 and/or $3(\sim 129$ and $\sim 128 \mathrm{kDa}$, respectively, Figure 3(a), top panel, denoted with a red dot), and the bottom band may represent variant $4(\sim 126 \mathrm{kDa}$, Figure 3(a), top panel, marked with a green dot). The cartoon shown in the bottom panel of Figure 3(a) illustrates a representative image of several western blots, analyzed at varying exposure times. The dotted lines indicate immunoreactive bands that only become evident after periods of long exposure.

Consistent with the RT-PCR data, our immunoblots also demonstrated the presence of at least one form of MyBP-C slow in each skeletal muscle tested. EDL and gastrocnemious possess three immunoreactive bands with the most prominent being the middle one (Figure 3(a), lanes 1 and 4). FDB also contains three distinct bands, however, the top and middle bands appear to be of similar intensities and occasionally appear as a broad, single band (Figure 3(a), lane 2). Similar to FDB, TA also shows two closely migrating bands, but lacks the bottom one (Figure 3(a) lane 3). Contrary to the rest of the muscles analyzed, quadriceps 


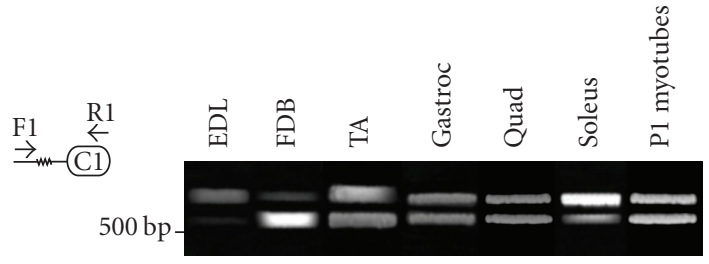

MyBP-C slow $\mathrm{NH}_{2}$-terminal insert

(a)

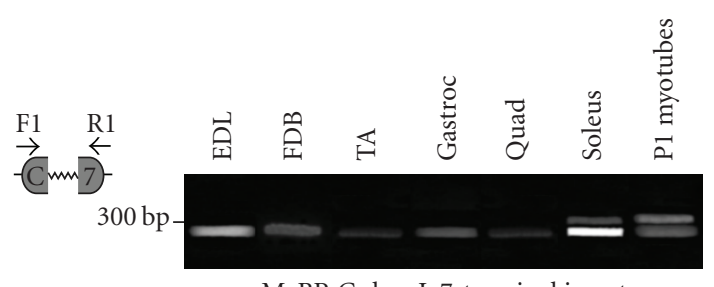

MyBP-C slow Ig7-terminal insert

(b)

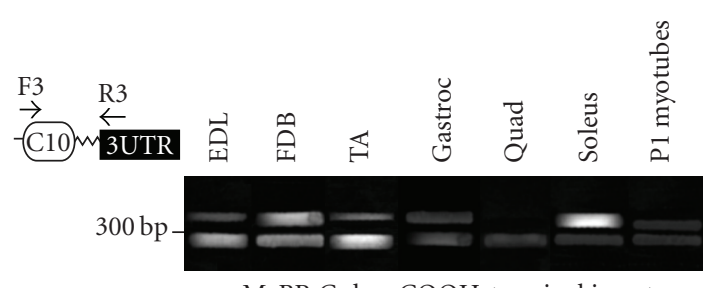

MyBP-C slow $\mathrm{COOH}$-terminal insert

(c)

FIGURE 2: RT-PCR analysis using cDNA generated from developing and adult rat extensor digitorum longus (EDL), flexor digitorum brevis (FDB), tibialis anterior (TA), gastrocnemius (gastroc), quadriceps (quad) and soleus skeletal muscles and primer sets designed to flank the $\mathrm{NH}_{2}$-terminal (a), Ig7 (b), and $\mathrm{COOH}$ terminal (c) insertions. A schematic representation of the amplified region is shown in the upper left corner of each panel. White and grey ovals depict Ig and FN-III domains, respectively, while the $3^{\prime}$ UTR is shown as a black line. The zig-zag lines denote the three novel insertions. In each amplification reaction, the top band corresponds to transcripts that include the insertion of interest, whereas the bottom band corresponds to transcripts that lack it. (a): All skeletal muscles tested contain a mixed population of MyBP-C slow variants that contain and lack the $\mathrm{NH}_{2}$-terminal insertion, as indicated by the presence of two amplification products. (b): On the contrary, only soleus and developing myotubes express transcripts that include the Ig7 insertion. (c): With the exception of quadriceps muscle that only contains $\mathrm{MyBP}-\mathrm{C}$ slow variants that lack the $\mathrm{COOH}$-terminal insertion, all other muscles examined contain a mixed pool of transcripts that carry and lack the novel $\mathrm{COOH}$ terminal insertion.

appears to contain only the middle band, although, the bottom one becomes evident after long exposure times (Figure 3(a) lane 5). Soleus expresses high amounts of the upper and lower bands, but moderate to low amounts of the middle one (Figure 3(a) lane 6). Finally, developing P1 skeletal myotubes show only one immunoreactive band with the same mobility as the top one, however, following longer exposure, the middle immunoreactive band is also apparent (Figure 3(a) lane 7).
TABle 1: Tabulated summary of the RT-PCR data shown in Figure 2. The plus $(+)$ and minus $(-)$ signs denote the presence or absence of mRNAs encoding the three regions of interest, respectively; the relative abundance of the respective transcripts is illustrated by the number of plus signs.

\begin{tabular}{lccc}
\hline Muscle Type & NH2-insert & Ig7-insert & COOH-insert \\
\hline Extensor Digitorum Longus & ++ & - & + \\
Flexor Digitorum Brevis & + & - & ++ \\
Tibialis Anterior & +++ & - & + \\
Gastrocnemius & ++ & - & + \\
Quadriceps & ++ & - & - \\
Soleus & ++++ & + & +++ \\
P1 Myotubes & +++ & ++ & + \\
\hline
\end{tabular}

We also probed the same muscle homogenates with an antibody that is specific for the $\mathrm{COOH}$-terminal insert and therefore only recognizes MyBP-C slow variant 1 (Figure 3(b)). With the exception of quadriceps, all of the other muscles were immunopositive for variant 1 . This finding is in agreement with our immunoblot data shown in panel A, and the RT-PCR analysis shown in Figure 2(c), which revealed that quadriceps was the sole muscle to lack the top immunoreactive band (i.e., variant 1) and the COOH-terminal insert, respectively. Notably, MyBP-C slow variant 1 appears to be expressed more abundantly in soleus muscle and least prominently in developing myotubes, while the remaining muscles tested contained intermediate amounts. The slight differences in the sizes of the bands detected in the different muscles are likely due to posttranslation modifications, as it has previously been shown that MyBP-C slow is capable of phosphorylation [37, 38].

Taken together, our RT-PCR and immunoblotting data (summarized in Table 2) clearly indicate that all skeletal muscles tested, apart from quadriceps, express variant 1 , albeit to different extents, with soleus and FDB containing the highest amounts. Variants 2 and 3 have complementary expression profiles, with variant 2 being preferentially expressed in muscles that have a higher composition of fast twitch fibers, such as EDL, FDB, TA, gastrocnemious and quadriceps, and variant 3 being selectively present in slow twitch muscles, like soleus, and developing myotubes. Finally, variant 4 is detected in all muscles examined, with the exception of TA and developing myotubes. It therefore appears that different forms of MyBP-C slow are present within the same muscle, independently of its fiber type composition, developmental stage, or age.

3.2. $M y B P-C$ at the A-band. Early studies have confirmed the direct interaction between MyBP-C and myosin and identified the sites of binding on both proteins. The light meromyosin (LMM) region of the myosin rod, that forms the backbone of the thick filament, binds the $\mathrm{COOH}$ terminal C10 region of all three MyBP-C isoforms [39-41]. Positively charged residues on the surface of the C10 domain mediate binding to LMM [42], however, the interaction is significantly strengthened by the presence of the C8 


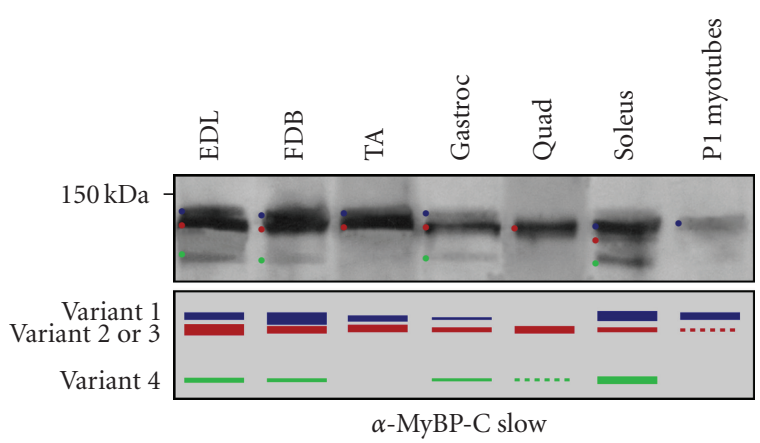

(a)

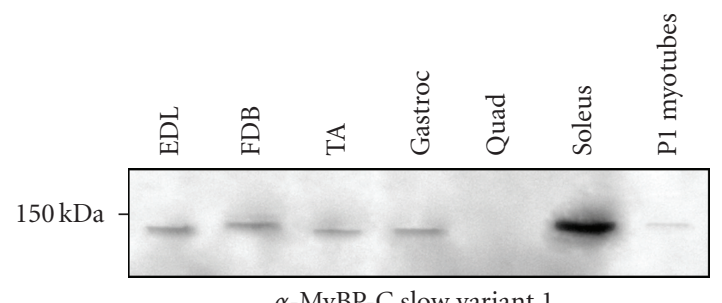

$\alpha$-MyBP-C slow variant 1

(b)

FIGURE 3: Western blot analysis of protein homogenates prepared from rat skeletal muscles and blotted with antibodies to the fifth Ig domain that recognize all MyBP-C slow variants (a) or to the novel $\mathrm{COOH}$-terminal insertion that specifically recognizes variant 1 (b). (a) Top panel: With the exception of quadriceps, all other muscles express variant 1 , represented by the upper most band, marked by a blue dot. All adult skeletal muscles tested, but not the developing myofibers, express variants 2 and/or 3, corresponding to the band with the intermediate mobility, and denoted with a red dot. As variants 2 and 3 have similar molecular weights $(129$ and $128 \mathrm{kDa}$, respectively), it is not feasible to separate them in the current gel system. Moreover, variant 4 is detected in homogenates prepared from EDL, FDB, gastrocnemious, and soleus, and is represented by the lower immunoreactive band, marked with a green dot. Bottom panel: A cartoon showing the presence of the different MyBP-C slow variants in developing and adult skeletal muscles. Dotted lines correspond to immunoreactive bands, which are evident only after long exposure times. (b) In agreement with the immunoblot shown in panel (a), antibodies specific for the novel $\mathrm{COOH}$-terminal insert demonstrated that EDL, FDB, TA, gastrocnemious, soleus, and P1 skeletal myotubes express variant 1 , with soleus containing the highest amounts.

and $\mathrm{C} 9$ regions. Another interaction between the MyBP$\mathrm{C}$ motif, located at the $\mathrm{NH}_{2}$-terminus of MyBP-C and subfragment 2 (S2) of myosin has been also identified, albeit of weaker affinity [43]. In addition to binding myosin, MyBP$\mathrm{C}$ associates with actin, in a $\mathrm{Ca}^{2+}$ dependent manner, through its $\mathrm{NH}_{2}$-terminal proline/alanine rich motif [44-46]. The ability of MyBP-C to directly interact with both myosin and actin facilitates its role as a regulator of cross-bridge formation during contraction. Interestingly, at low ionic strength MyBP-C inhibits actomyosin ATPase activity, while at higher ionic strength it acts as a mild activator $[47,48]$.

In addition to myosin, MyBP-C also associates with titin at the $A$-band $[14,49-51]$. The COOH-terminal C8-C10
TABLE 2: MyBP-C slow variants 1-4 are present in varying amounts in different skeletal muscles. A plus (+) sign indicates the presence of the respective variant in a select muscle. The relative abundance of variants 1-4 in the skeletal muscles tested is illustrated by the number of plus signs.

\begin{tabular}{|c|c|c|c|c|}
\hline Muscle Type & Variant 1 & Variant 2 & Variant 3 & Variant 4 \\
\hline $\begin{array}{l}\text { Extensor Digitorum } \\
\text { Longus }\end{array}$ & ++ & +++ & - & ++ \\
\hline $\begin{array}{l}\text { Flexor Digitorum } \\
\text { Brevis }\end{array}$ & +++ & ++ & 一 & ++ \\
\hline Tibialis Anterior & ++ & ++ & - & - \\
\hline Gastrocnemius & + & + & - & + \\
\hline Quadriceps & - & ++ & - & - \\
\hline Soleus & ++++ & - & + & +++ \\
\hline P1 Myotubes & ++ & 一 & + & - \\
\hline
\end{tabular}

domains of MyBP-C directly bind to the 11-domain superrepeat $\left[\mathrm{Ig}-(\mathrm{FN}-\mathrm{III})_{2}-\mathrm{Ig}-(\mathrm{FN}-\mathrm{III})_{3}-\mathrm{Ig}-(\mathrm{FN}-\mathrm{III})_{3}\right]$ present in the $\mathrm{C}$-zone portion of titin, and specifically the first Ig domain, although flanking motifs further strengthen the interaction [52, 53]. Notably, the arrangement of MyBP$\mathrm{C}$ in 11 transverse stripes at regular intervals of $\sim 43 \mathrm{~nm}$ corresponds to that of the 11-domain super-repeat of titin, to which MyBP-C binds. Consequently, It has been suggested that binding to titin's super-repeats specifies the subsarcomeric distribution of MyBP-C in the C-zone of the A-band [52].

Recent studies have proposed that MyBP-C forms a trimeric "collar" around each thick filament in which domains C5-C7 of one molecule overlap with domains C8$\mathrm{C} 10$ of the neighboring molecule $[54,55]$. In vitro binding studies using the respective peptides postulated that this arrangement might apply to the cardiac and fast skeletal isoforms, but not the slow isoform. Interestingly, for these studies Flashman and coworkers generated a recombinant protein that contained domains $\mathrm{C} 8-\mathrm{C} 10$, present in all four slow variants, followed by the novel COOH-terminal insertion, present only in variant 1 . It is, therefore, possible that the presence of the $\mathrm{COOH}$-terminal 26-amino acids may inhibit binding of the $\mathrm{C} 8-\mathrm{C} 10$ domains of variant 1 to the C5-C7 domains of the neighboring molecule of MyBP-slow. Indeed, this is consistent with our recent studies indicating that variant 1 is preferentially localized at the periphery of the $M$-band [28]. Conversely, an interaction between the respective motifs present in variants $2-4$ is likely, as these contain a short $\mathrm{COOH}$-terminus following domain $\mathrm{C} 10$ that, similar to the cardiac and fast isoforms, consists of four amino acids.

Through its interactions with myosin and titin, MyBP$\mathrm{C}$ contributes to the stabilization and maintenance of the sarcomeric $A$-band. In vitro studies indicate that myosin filaments are capable of forming in the absence of MyBP$\mathrm{C}$, however, its addition results in increased filament length, and improved structure and uniformity across the filament $[40,56,57]$. Additionally, in vivo deletion of the myosin 


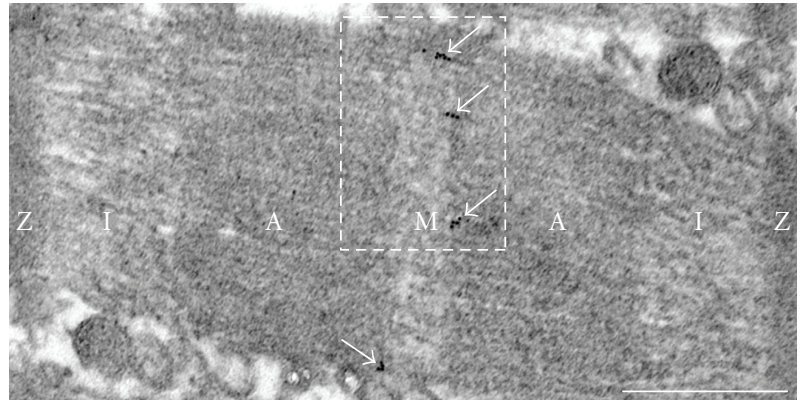

(a)

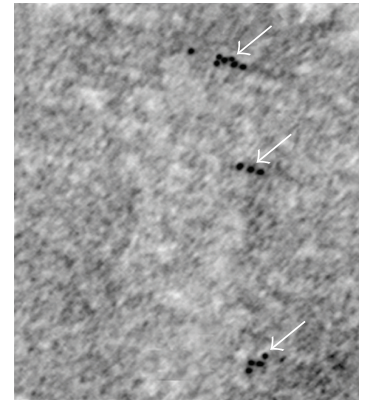

(b)

FIGURE 4: Ultrathin cryo-sections of adult mouse FDB skeletal muscle were labeled with antibodies specific for the COOH-terminal insertion present in MyBP-C slow variant 1. (a) Variant 1 was detected at the periphery of the $M$-band (arrows). The boxed area in (a) is blown up in (b) for ease of visualization of the immunolabeling. Scale bar corresponds to $0.5 \mu \mathrm{m}$.

and titin binding sites on MyBP-C results in disorganized $A$ bands $[58,59]$. Consistent with this, the C8-C10 domains that harbor the binding sites for myosin and titin are deleted in patients suffering from familial hypertrophic cardiomyopathy $[3,60,61]$. Interestingly, though, normal A-bands are formed in animals deficient of cardiac MyBPC $[62,63]$, suggesting the presence of a compensatory mechanism that maintains the myosin and titin filaments within the $A$-band of the sarcomere. Taken together, these studies suggest that MyBP-C, through its direct interaction with myosin and titin, and possibly its self-association, may stabilize the sarcomeric $A$-band.

In addition to maintaining the structure of thick filaments, MyBP-C may also play key roles in regulating contractile function by modulating the rate of cross-bridges formation. Consistent with this, in cardiomyocytes lacking MyBP-C, actomyosin filaments exhibited increased power output during contraction and faster rates of force development at half maximal $\mathrm{Ca}^{2+}$ activation [64]. Reintroduction of the $\mathrm{NH}_{2}$-terminal region of cardiac MyBP-C, containing the myosin S2 binding site (i.e., domains $\mathrm{C} 1$ and $\mathrm{C} 2$ ), enhanced $\mathrm{Ca}^{2+}$ sensitivity and restored the contractile properties of the null cardiocytes to normal levels [65], suggesting that cardiac MyBP-C contributes to the regulation of myofilament tension and their cycling rates.

Contrary to the cardiac isoform that has been directly implicated in the regulation of the contractile properties of cardiomyocytes (reviewed in [3]), studies focusing on the function of the skeletal forms of MyBP-C are limited. However, early studies have suggested that the role of MyBPC in skeletal muscle likely parallels that of the cardiac isoform [66]. Consistent with this, the amounts of MyBP-C slow were recently found significantly increased in EDL muscles from a mouse model in which the kinase domain of titin was deleted [67]. Deficient EDL muscles exhibited reduced myofilament $\mathrm{Ca}^{2+}$ sensitivity and altered contractile properties, which were restored to normal levels upon extraction of MyBP-C slow. Contrary to the slow form of MyBP-C, the fast form was significantly downregulated in the same muscles, while the expression levels of other proteins of the $A$ - and $M$ bands remained unaffected. It is, therefore, likely that the kinase domain of titin affects the expression of genes involved in the regulation of myofilament $\mathrm{Ca}^{2+}$ sensitivity and force production. Experimental evidence has therefore started to emerge suggesting a key role for the skeletal forms of MyBP$\mathrm{C}$ in modulating contractility, too.

\subsection{Mybp-C Slow Variant 1 Selectively Concentrates at the M-}

Band. The presence of MyBP-C slow at the C-zones of the $A$-band has been studied extensively during the last three decades $[3,26,68]$. Recent studies from our laboratory, however, have provided evidence that at least one form of MyBP-C slow, specifically variant 1 , has a unique topography in the muscle cell [28]. Detailed immunofluorescence studies combined with confocal microscopy demonstrated that MyBP-C slow variant 1 is selectively localized at the periphery of the $M$-band in adult rat soleus muscle [28]. The unique localization of MyBP-C slow variant 1 is further supported by our ultrastructural studies, shown in Figure 4 . Immunolabeling of ultrathin cryosections prepared from adult mouse FDB muscle with antibodies to the unique $\mathrm{COOH}$-terminus of variant 1 also demonstrated that it preferentially concentrates at the edges of the sarcomeric $M$-band (Figures 4(a)-4(b), arrowheads). Thus, it appears that the different MyBP-C slow isoforms have distinct distributions in skeletal myofibers, with variants $2-4$ localizing at the $A$ band and variant 1 at the $M$-band.

At the $M$-band, MyBP-C slow variant 1 interacts with obscurin and four and a half lim protein 1 (FHL1) $[28,69]$. The second Ig domain of obscurin and the last Ig domain of MyBP-C slow variant 1 are both necessary and sufficient to support their interaction, however, binding is enhanced significantly in the presence of the novel 26 amino acids at the $\mathrm{COOH}$-terminus of variant 1 . Overexpression of the second Ig domain of obscurin in primary cultures of skeletal myotubes inhibited the integration of MyBP-C slow variant 1 at the $M$-band and severely disrupted the formation of $M$ - and $A$-bands. These findings suggested that variant 1 might contribute to the assembly and integrity of these structures via its interaction with obscurin and possibly other proteins. Similarly, McGrath et al. also demonstrated 
that overexpression or downregulation of FHL1, which is localized at the $A$-I junction and the $M$-band, in adult mouse soleus muscle resulted in impaired thick filament assembly, which was accompanied by reduced sarcomeric incorporation of different forms of MyBP-C slow, including variant $1[69]$.

3.4. MyBP-C Slow in Muscle Disease. Mutations within the cardiac isoform of MyBP-C cause familial hypertrophic cardiomyopathy (reviewed in $[70,71])$. To date, there are no muscle diseases causally associated with mutations in the transcripts of the skeletal forms of MyBP-C. However, changes in the expression levels of the skeletal isoforms have been reported in hypertrophic and dystrophic skeletal muscles. The expression levels of MyBP-C slow were significantly increased in rat plantaris muscle induced to hypertrophy by surgical ablation of the neighboring soleus and gastrocnemius muscles, whereas the amounts of MyBPC fast were dramatically decreased [72]. Likewise, the protein levels of MyBP-C slow were increased in both chicken and mouse dystrophic skeletal muscles [20, 24]. Further research is required in order to understand the molecular mechanisms that lead to the differential regulation of the skeletal forms of MyBP-C in these models, and their potentially unique roles in regulating the formation and activity of contractile structures.

\section{Summary and Future Perspectives}

MyBP-C slow comprises a subfamily of at least four isoforms that result from complex alternative splicing of the single MyBP-C slow gene. The presence of the four MyBP-C slow variants is not restricted to slow-twitch muscles, as select isoforms are abundantly expressed in fast-twitch muscles, too, where they may coexist with MyBP-C fast. More importantly, the four isoforms of MyBP-C slow may be coexpressed in the same muscle, fiber or sarcomere, where they may exhibit unique topographies concentrating either at $A$-bands (variants 2, 3, and 4) or $M$-bands (variant 1). Previous and current findings strongly favor a structural role for MyBP$\mathrm{C}$ slow at the sarcomeric $A$ - and $M$-bands, and point to a regulatory role on muscle contraction. Consequently, the detailed characterization of the biochemical and biophysical properties of the four MyBP-C slow variants, and the development of the appropriate molecular tools and animal models are imperative in order to study the cell biology and functional properties of this intricate subfamily of proteins.

\section{Acknowledgments}

The authors wish to thank S. Adediran and N. Perry for their technical assistance in the initial stages of this work. Our research has been supported by Grants to Aikaterini Kontrogianni-Konstantopoulos from the National Institutes of Health (R01 AR52768) and the Muscular Dystrophy Association (RG 4214) and to Maegen A. Ackermann from the National Institutes of Health (F32 AR058079).

\section{References}

[1] K. A. Clark, A. S. McElhinny, M. C. Beckerle, and C. C. Gregorio, "Striated muscle cytoarchitecture: an intricate web of form and function," Annual Review of Cell and Developmental Biology, vol. 18, pp. 637-706, 2002.

[2] C. Franzini-Armstrong, "Functional significance of membrane architecture in skeletal and cardiac muscle," Society of General Physiologists series, vol. 51, pp. 3-18, 1996.

[3] C. E. Oakley, J. Chamoun, L. J. Brown, and B. D. Hambly, "Myosin binding protein-C: enigmatic regulator of cardiac contraction," International Journal of Biochemistry and Cell Biology, vol. 39, no. 12, pp. 2161-2166, 2007.

[4] R. Starr and G. Offer, "Polypeptide chains of intermediate molecular weight in myosin preparations," FEBS Letters, vol. 15 , no. 1 , pp. 40-44, 1971.

[5] H. C. Hartzell and W. S. Sale, "Structure of C protein purified from cardiac muscle," Journal of Cell Biology, vol. 100, no. 1, pp. 208-215, 1985.

[6] G. Offer, C. Moos, and R. Starr, "A new protein of the thick filaments of vertebrate skeletal myofibrils. Extraction, purification and characterization," Journal of Molecular Biology, vol. 74, no. 4, pp. 653-662, 1973.

[7] R. Craig and G. Offer, "The location of C protein in rabbit skeletal muscle," Proceedings of the Royal Society of London. Series B, vol. 192, no. 1109, pp. 451-461, 1976.

[8] P. Bennett, R. Craig, R. Starr, and G. Offer, "The ultrastructural location of C-protein, X-protein and $\mathrm{H}$-protein in rabbit muscle," Journal of Muscle Research and Cell Motility, vol. 7, no. 6 , pp. 550-567, 1986.

[9] F. A. Pepe, "Structure of muscle filaments from immunohistochemical and ultrastructural studies," Journal of Histochemistry and Cytochemistry, vol. 23, no. 7, pp. 543-562, 1975.

[10] F. A. Pepe, F. T. Ashton, C. Street, and J. Weisel, "The myosin filament. X. Observation of nine subfilaments in transverse sections," Tissue and Cell, vol. 18, no. 4, pp. 499-508, 1986.

[11] E. Rome, G. Offer, and F. A. Pepe, "X ray diffraction of muscle labelled with antibody to C protein," Nature: New Biology, vol. 244, no. 135, pp. 152-154, 1973.

[12] M. Gautel, M. A. C. Morelli, M. Pfuhl, A. Motta, and A. Pastore, "A calmodulin-binding sequence in the C-terminus of human cardiac titin kinase," European Journal of Biochemistry, vol. 230, no. 2, pp. 752-759, 1995.

[13] F. E. Weber, K. T. Vaughan, F. C. Reinach, and D. A. Fischman, "Complete sequence of human fast-type and slow-type muscle myosin-binding-protein C (MyBP-C). Differential expression, conserved domain structure and chromosome assignment," European Journal of Biochemistry, vol. 216, no. 2, pp. 661-669, 1993.

[14] D. O. Fürst, U. Vinkemeier, and K. Weber, "Mammalian skeletal muscle C-protein: purification from bovine muscle, binding to titin and the characterization of a full-length human cDNA," Journal of Cell Science, vol. 102, no. 4, pp. 769778, 1992.

[15] F. C. Reinach, T. Masaki, and D. A. Fischman, "Characterization of the C-protein from posterior latissimus dorsi muscle of the adult chicken: heterogeneity within a single sarcomere," Journal of Cell Biology, vol. 96, no. 1, pp. 297-300, 1983.

[16] M. Yasuda, S. Koshida, N. Sato, and T. Obinata, "Complete primary structure of chicken cardiac C-protein (MyBP-C) and its expression in developing striated muscles," Journal of Molecular and Cellular Cardiology, vol. 27, no. 10, pp. 22752286, 1995. 
[17] S. Einheber and D. A. Fischman, "Isolation and characterization of a cDNA clone encoding avian skeletal muscle Cprotein: an intracellular member of the immunoglobulin superfamily," Proceedings of the National Academy of Sciences of the United States of America, vol. 87, no. 6, pp. 2157-2161, 1990.

[18] M. Gautel, D. O. Fürst, A. Cocco, and S. Schiaffino, "Isoform transitions of the myosin binding protein $\mathrm{C}$ family in developing human and mouse muscles: lack of isoform transcomplementation in cardiac muscle," Circulation Research, vol. 82, no. 1, pp. 124-129, 1998.

[19] M. Kawashima, S. Kitani, T. Tanaka, and T. Obinata, "The earliest form of C-protein expressed during striated muscle development is immunologically the same as cardiac-type Cprotein," Journal of Biochemistry, vol. 99, no. 4, pp. 1037-1047, 1986.

[20] M. Kurasawa, N. Sato, A. Matsuda, S. Koshida, T. Totsuka, and T. Obinata, "Differential expression of C-protein isoforms in developing and degenerating mouse striated muscles," Muscle and Nerve, vol. 22, no. 2, pp. 196-207, 1999.

[21] T. Obinata, F. C. Reinach, D. M. Bader, T. Masaki, S. Kitani, and D. A. Fischman, "Immunochemical analysis of C-protein isoform transitions during the development of chicken skeletal muscle," Developmental Biology, vol. 101, no. 1, pp. 116-124, 1984.

[22] F. Fougerousse, A.-L. Delezoide, M. Y. Fiszman, K. Schwartz, J. S. Beckmann, and L. Carrier, "Cardiac myosin binding protein $\mathrm{C}$ gene is specifically expressed in heart during murine and human development," Circulation Research, vol. 82, no. 1, pp. 130-133, 1998.

[23] T. Obinata, S. Kitani, T. Masaki, and D. A. Fischman, "Coexistence of fast-type and slow-type C-proteins in neonatal chicken breast muscle," Developmental Biology, vol. 105, no. 1, pp. 253-256, 1984.

[24] T. Obinata and K. Shinbo, "Slow-type C-protein in dystrophic chicken fast pectoralis muscle," Muscle and Nerve, vol. 10, no. 4, pp. 351-358, 1987.

[25] G. K. Dhoot and S. V. Perry, "Expression of slow skeletal myosin binding C-protein in normal adult mammalian heart," Journal of Muscle Research and Cell Motility, vol. 26, no. 2, pp. 143-148, 2005.

[26] L. Carrier, "Cardiac myosin-binding protein C in the heart," Archives des Maladies du Coeur et des Vaisseaux, vol. 100, no. 3, pp. 238-243, 2007.

[27] P. P. de Tombe, "Myosin binding protein $\mathrm{C}$ in the heart," Circulation Research, vol. 98, no. 10, pp. 1234-1236, 2006.

[28] M. A. Ackermann, L.-Y. R. Hu, A. L. Bowman, R. J. Bloch, and A. Kontrogianni-Konstantopoulos, "Obscurin interacts with a novel isoform of MyBP-C slow at the periphery of the sarcomeric M-band and regulates thick filament assembly," Molecular Biology of the Cell, vol. 20, no. 12, pp. 2963-2978, 2009.

[29] A. Kontrogianni-Konstantopoulos and R. J. Bloch, "Obscurin: a multitasking muscle giant," Journal of Muscle Research and Cell Motility, vol. 26, no. 6-8, pp. 419-426, 2005.

[30] S. V. Yap, E. Vafiadaki, J. Strong, and A. KontrogianniKonstantopoulos, "HAX-1: a multifaceted antiapoptotic protein localizing in the mitochondria and the sarcoplasmic reticulum of striated muscle cells," Journal of Molecular and Cellular Cardiology. In press.

[31] J. Lexell, J. C. Jarvis, J. Currie, D. Y. Downham, and S. Salmons, "Fibre type composition of rabbit tibialis anterior and extensor digitorum longus muscles," Journal of Anatomy, vol. 185, no. 1, pp. 95-101, 1994.
[32] T. Soukup, G. Zacharova, and V. Smerdu, "Fibre type composition of soleus and extensor digitorum longus muscles in normal female inbred Lewis rats," Acta Histochemica, vol. 104, no. 4, pp. 399-405, 2002.

[33] E. Gonzalez, M. L. Messi, Z. Zheng, and O. Delbono, "Insulinlike growth factor-1 prevents age-related decrease in specific force and intracellular $\mathrm{Ca}^{2+}$ in single intact muscle fibres from transgenic mice," Journal of Physiology, vol. 552, no. 3, pp. 833844, 2003.

[34] N. Hamalainen and D. Pette, "The histochemical profiles of fast fiber types IIB, IID, and IIA in skeletal muscles of mouse, rat, and rabbit," Journal of Histochemistry and Cytochemistry, vol. 41, no. 5, pp. 733-743, 1993.

[35] P. D. Gollnick, B. Sjoedin, J. Karlsson, E. Jansson, and B. Saltin, "Human soleus muscle: a comparison of fiber composition and enzyme activities with other leg muscles," Pflugers Archiv European Journal of Physiology, vol. 348, no. 3, pp. 247-255, 1974.

[36] A. R. Tupling, E. Bombardier, R. D. Stewart, C. Vigna, and A. E. Aqui, "Muscle fiber type-specific response of Hsp70 expression in human quadriceps following acute isometric exercise," Journal of Applied Physiology, vol. 103, no. 6, pp. 2105-2111, 2007.

[37] M. S. Lim and M. P. Walsh, "Phosphorylation of skeletal and cardiac muscle C-proteins by the catalytic subunit of cAMPdependent protein kinase," Biochemistry and Cell Biology, vol. 64, no. 7, pp. 622-630, 1986.

[38] I. M. Vikhliantsev and Z. A. Podlubnaia, "Phosphorylation of sarcomeric cytoskeletal proteins-an adaptive factor for inhibiting the contractile activity of muscle during hibernation," Biofizika, vol. 48, no. 3, pp. 499-504, 2003.

[39] T. N. Alyonycheva, T. Mikawa, F. C. Reinach, and D. A. Fischman, "Isoform-specific interaction of the myosinbinding proteins (MyBPs) with skeletal and cardiac myosin is a property of the C-terminal immunoglobulin domain," Journal of Biological Chemistry, vol. 272, no. 33, pp. 20866-20872, 1997.

[40] C. Moos, G. Offer, R. Starr, and P. Bennett, "Interaction of C protein with myosin, myosin rod and light meromyosin," Journal of Molecular Biology, vol. 97, no. 1, pp. 1-9, 1975.

[41] T. Okagaki, F. E. Weber, D. A. Fischman, K. T. Vaughan, T. Mikawa, and F. C. Reinach, "The major myosin-binding domain of skeletal muscle MyBP-C (C protein) resides in the COOH-terminal, immunoglobulin C2 motif," Journal of Cell Biology, vol. 123, no. 3, pp. 619-626, 1993.

[42] C. A. Miyamoto, D. A. Fischman, and F. C. Reinach, "The interface between MyBP-C and myosin: site-directed mutagenesis of the CX myosin-binding domain of MyBP-C," Journal of Muscle Research and Cell Motility, vol. 20, no. 7, pp. 703715, 1999.

[43] R. Starr and G. Offer, "The interaction of C-protein with heavy meromyosin and subfragment-2," Biochemical Journal, vol. 171, no. 3, pp. 813-816, 1978.

[44] C. Moos, "Fluorescence microscope study of the binding of added C protein to skeletal muscle myofibrils," Journal of Cell Biology, vol. 90, no. 1, pp. 25-31, 1981.

[45] C. Moos, C. M. Mason, and J. M. Besterman, "The binding of skeletal muscle C-protein to F-actin, and its relation to the interaction of actin with myosin subfragment-1," Journal of Molecular Biology, vol. 124, no. 5, pp. 571-586, 1978.

[46] K. Yamamoto, "The binding of skeletal muscle C-protein to regulated actin," FEBS Letters, vol. 208, no. 1, pp. 123-127, 1986. 
[47] N. A. Freydina, Z. I. Vishnevskaya, S. N. Udaltsov, and Z. A. Podlubnaya, "Effect of C-protein and LC-light chains on actomyosin ATPase at various ionic strength and calcium levels," Acta Biochimica et Biophysica Hungarica, vol. 21, no. 3, pp. 247-256, 1986.

[48] C. Moos and I. N. Feng, "Effect of C-protein on actomyosin ATPase," Biochimica et Biophysica Acta, vol. 632, no. 2, pp. 141-149, 1980.

[49] J. F. Koretz, T. C. Irving, and K. Wang, "Filamentous aggregates of native titin and binding of C-protein and AMP-deaminase," Archives of Biochemistry and Biophysics, vol. 304, no. 2, pp. 305-309, 1993.

[50] S. Labeit, M. Gautel, A. Lakey, and J. Trinick, "Towards a molecular understanding of titin," The EMBO Journal, vol. 11, no. 5, pp. 1711-1716, 1992.

[51] A. Soteriou, M. Gamage, and J. Trinick, "A survey of interactions made by the giant protein titin," Journal of Cell Science, vol. 104, no. 1, pp. 119-123, 1993.

[52] A. Freiburg and M. Gautel, "A molecular map of the interactions between titin and myosin-binding protein $\mathrm{C}$ : implications for sarcomeric assembly in familial hypertrophic cardiomyopathy," European Journal of Biochemistry, vol. 235, no. 1-2, pp. 317-323, 1996.

[53] A. Kontrogianni-Konstantopoulos, M. A. Ackermann, A. L. Bowman, S. V. Yap, and R. J. Bloch, "Muscle giants: molecular scaffolds in sarcomerogenesis," Physiological Reviews, vol. 89, no. 4, pp. 1217-1267, 2009.

[54] E. Flashman, L. Korkie, H. Watkins, C. Redwood, and J. C. Moolman-Smook, "Support for a trimeric collar of myosin binding protein $\mathrm{C}$ in cardiac and fast skeletal muscle, but not in slow skeletal muscle," FEBS Letters, vol. 582, no. 3, pp. 434438, 2008.

[55] B. Decker and M. S. Z. Kellermayer, "Periodically arranged interactions within the myosin filament backbone revealed by mechanical unzipping," Journal of Molecular Biology, vol. 377, no. 2, pp. 307-310, 2008.

[56] J. S. Davis, "Interaction of C-protein with $\mathrm{pH} 8.0$ synthetic thick filaments prepared from the myosin of vertebrate skeletal muscle," Journal of Muscle Research and Cell Motility, vol. 9, no. 2, pp. 174-183, 1988.

[57] J. F. Koretz, "Effects of C-protein on synthetic myosin filament structure," Biophysical Journal, vol. 27, no. 3, pp. 433-446, 1979.

[58] R. Gilbert, J. A. Cohen, S. Pardo, A. Basu, and D. A. Fischman, "Identification of the A-band localization domain of myosin binding proteins $\mathrm{C}$ and $\mathrm{H}$ (MyBP-C, MyBP-H) in skeletal muscle," Journal of Cell Science, vol. 112, no. 1, pp. 69-79, 1999.

[59] R. Gilbert, M. G. Kelly, T. Mikawa, and D. A. Fischman, "The carboxyl terminus of myosin binding protein C (MyBP-C, Cprotein) specifies incorporation into the A-band of striated muscle," Journal of Cell Science, vol. 109, no. 1, pp. 101-111, 1996.

[60] G. Bonne, L. Carrier, J. Bercovici, et al., "Cardiac myosin binding protein-C gene splice acceptor site mutation is associated with familial hypertrophic cardiomyopathy," Nature Genetics, vol. 11, no. 4, pp. 438-440, 1995.

[61] C. E. Oakley, B. D. Hambly, P. M. Curmi, and L. J. Brown, "Myosin binding protein C: structural abnormalities in familial hypertrophic cardiomyopathy," Cell research, vol. 14, no. 2, pp. 95-110, 2004.

[62] L. Carrier, R. Knöll, N. Vignier, et al., "Asymmetric septal hypertrophy in heterozygous cMyBP-C null mice," Cardiovascular Research, vol. 63, no. 2, pp. 293-304, 2004.
[63] S. P. Harris, C. R. Bartley, T. A. Hacker, et al., "Hypertrophic cardiomyopathy in cardiac myosin binding protein-C knockout mice," Circulation Research, vol. 90, no. 5, pp. 594-601, 2002.

[64] F. S. Korte, K. S. McDonald, S. P. Harris, and R. L. Moss, "Loaded shortening, power output, and rate of force redevelopment are increased with knockout of cardiac myosin binding protein-C," Circulation Research, vol. 93, no. 8, pp. 752-758, 2003.

[65] S. P. Harris, E. Rostkova, M. Gautel, and R. L. Moss, "Binding of myosin binding protein-C to myosin subfragment $\mathrm{S} 2$ affects contractility independent of a tether mechanism," Circulation Research, vol. 95, no. 9, pp. 930-936, 2004.

[66] P. A. Hofmann, M. L. Greaser, and R. L. Moss, "C-protein limits shortening velocity of rabbit skeletal muscle fibres at low levels of $\mathrm{Ca}^{2+}$ activation," Journal of Physiology, vol. 439, pp. 701-715, 1991.

[67] C. A. C. Ottenheijm, C. Hidalgo, K. Rost, M. Gotthardt, and H. Granzier, "Altered contractility of skeletal muscle in mice deficient in titin's M-band region," Journal of Molecular Biology, vol. 393, no. 1, pp. 10-26, 2009.

[68] S. Winegrad, "Cardiac myosin binding protein C," Circulation Research, vol. 84, no. 10, pp. 1117-1126, 1999.

[69] M. J. McGrath, D. L. Cottle, M.-A. Nguyen, et al., "Four and a half LIM protein 1 binds myosin-binding protein $\mathrm{C}$ and regulates myosin filament formation and sarcomere assembly," Journal of Biological Chemistry, vol. 281, no. 11, pp. 76667683, 2006.

[70] E. Flashman, C. Redwood, J. Moolman-Smook, and H. Watkins, "Cardiac myosin binding protein C: its role in physiology and disease," Circulation Research, vol. 94, no. 10, pp. 1279-1289, 2004.

[71] D. Sanoudou, E. Vafiadaki, D. A. Arvanitis, E. Kranias, and A. Kontrogianni-Konstantopoulos, "Array lessons from the heart: focus on the genome and transcriptome of cardiomyopathies," Physiological Genomics, vol. 21, pp. 131-143, 2005.

[72] K. M. McCormick, K. M. Baldwin, and F. Schachat, "Coordinate changes in $\mathrm{C}$ protein and myosin expression during skeletal muscle hypertrophy," American Journal of Physiology, vol. 267, no. 2, pp. C443-C449, 1994. 

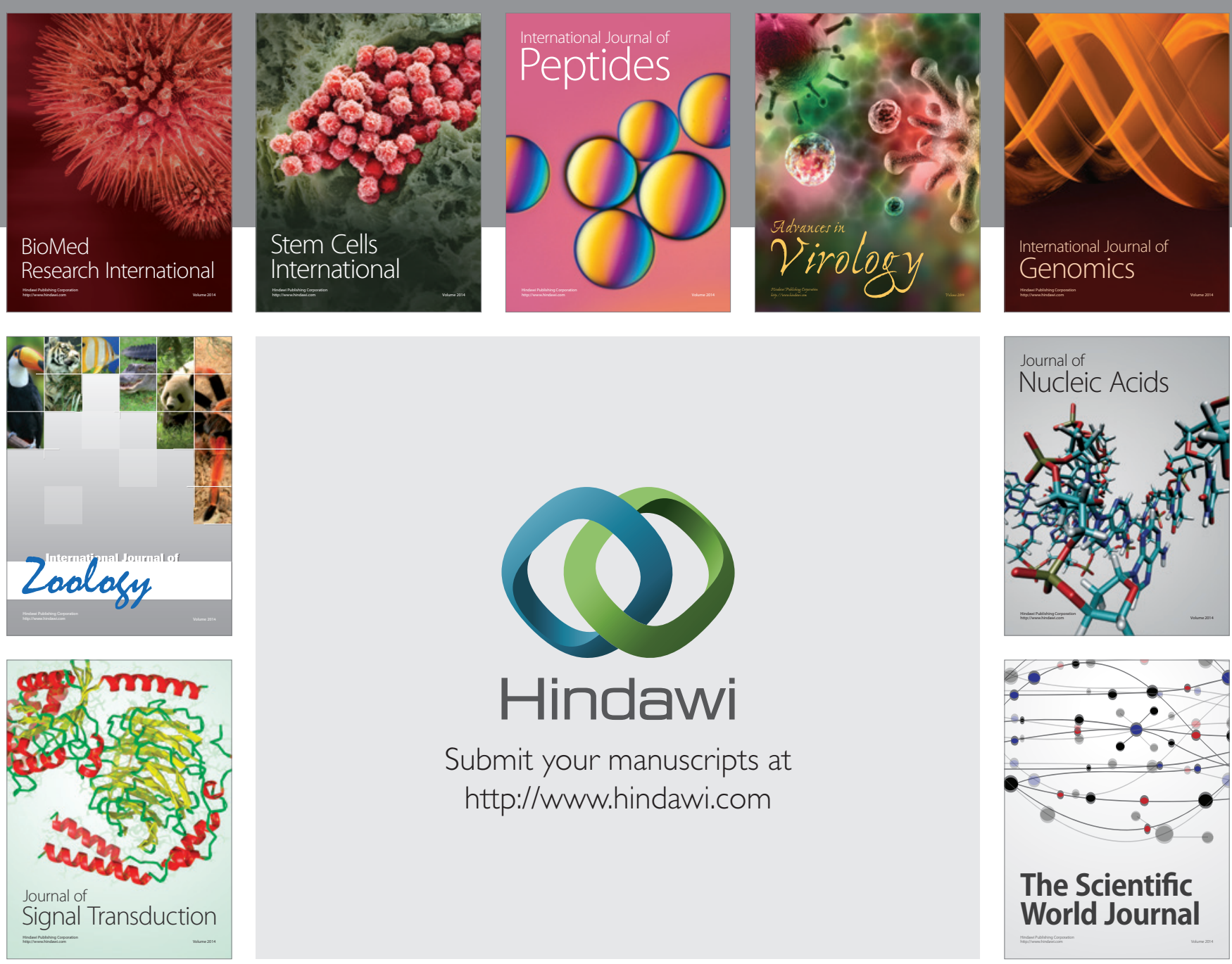

Submit your manuscripts at

http://www.hindawi.com
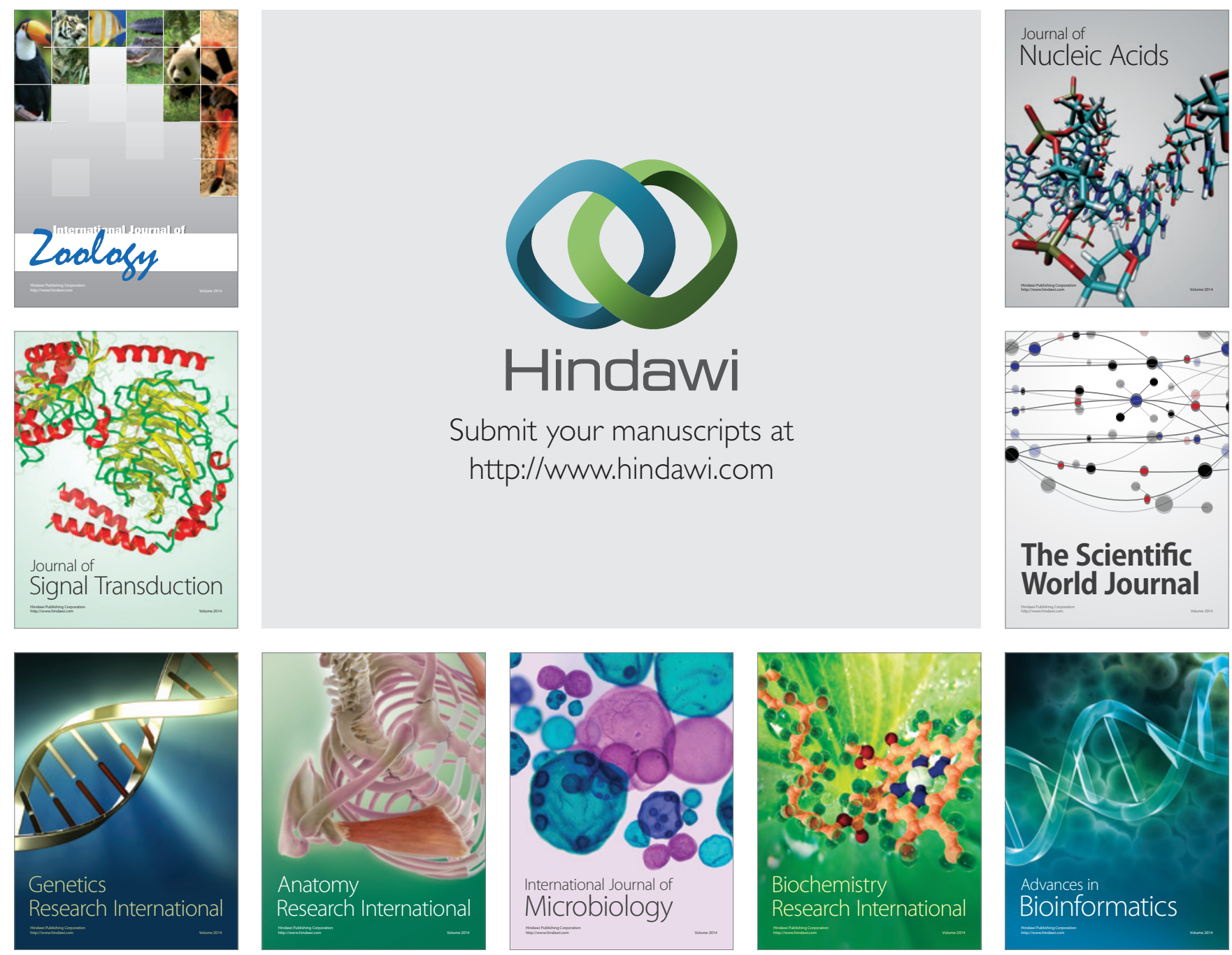

The Scientific World Journal
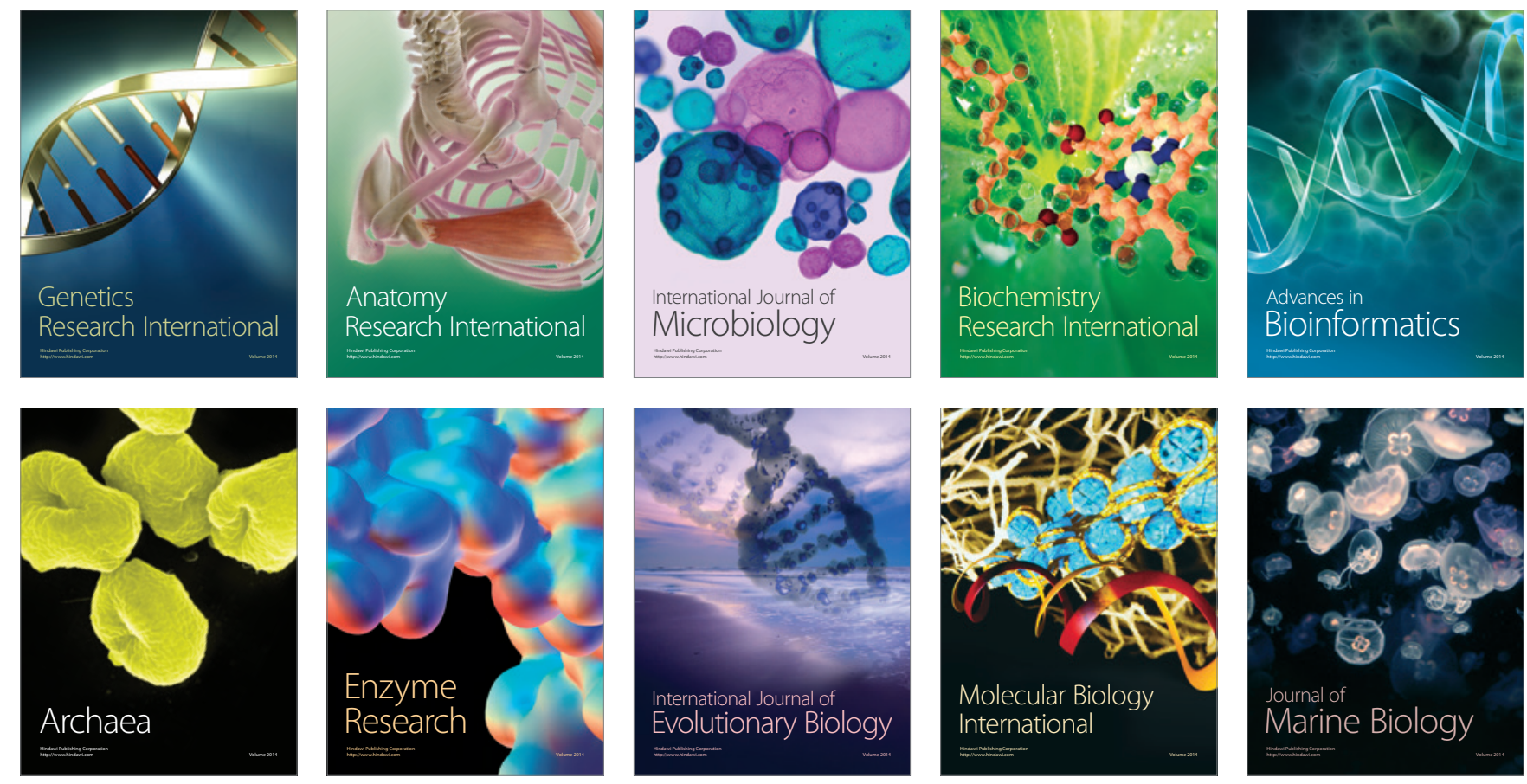